\title{
Forecasting the Performance of Hedge Fund Styles*
}

\author{
Jose Olmo, Centro Universitario de la Defensa and City University London \\ Marcos Sanso-Navarro† Universidad de Zaragoza
}

September 2011

\begin{abstract}
This article predicts the relative performance of hedge fund investment styles one period ahead using time-varying conditional stochastic dominance tests. These tests allow the construction of dynamic trading strategies based on nonparametric density forecasts of hedge fund returns. During the recent financial turmoil, our tests predict a superior performance of the Global Macro investment style compared to the other 'Directional Traders' strategies. The Dedicated Short Bias investment style is, on the other hand, stochastically dominated by the other directional styles. These results are confirmed by simple nonparametric tests constructed from the realized excess returns. Further, by exploiting the cross-validation method for optimal bandwidth parameter selection, we find out which factors have predictive power for the density of hedge fund returns. We observe that different factors have forecasting power for different regions of the returns distribution and, more importantly, Fung and Hsieh factors have power not only for describing the risk premium but also for density forecasting if appropriately exploited.
\end{abstract}

Keywords: Conditional density estimation, hedge fund styles, nonparametric methods, portfolio performance, stochastic dominance tests.

JEL codes: C1, C2, G1.

\footnotetext{
*The authors acknowledge financial support from Ministerio de Ciencia e Innovación (ECO2011-22650 project) and the Regional Government of Aragón (ADETRE Research Group).

${ }^{\dagger}$ Corresponding author. Address: Departamento de Análisis Económico. Facultad de Economía y Empresa. Gran Vía 2. 50005 Zaragoza (Spain). Phone: +34 876554 629. Fax: +34 976761996 e-mail: marcossn@unizar.es.
} 


\section{Introduction}

Hedge funds have attracted a great deal of attention during the last fifteen years. These financial instruments are private investment vehicles for wealthy individuals and institutional investors that are lightly regulated and supervised. Following unconventional trading strategies, these funds have traditionally outperformed the other investment strategies partly due to the weak correlation of their returns with those of other financial securities. This stylized fact has recently been disputed: the 2007-08 crisis has revealed the interdependencies of these funds with the rest of the financial industry.

The sequence of papers by Fung and Hsieh (1997, 2001, 2002, 2004a, 2004b) showed that the risk premium from these funds can be largely explained by a set of financial variables rather different from the standard capital asset pricing formulations widely used in the mutual fund investment literature. These findings are crucial for constructing optimal portfolios. Agarwal and Naik (2004) study the relative performance between hedge funds and also against mutual funds. Related articles are Capocci and Hübner (2004) and Eling and Faust (2010). Patton (2009) also contributed to the study of these investment vehicles by questioning their market neutrality.

Financial return predictability has a long tradition in the empirical finance literature, see Keim and Stambaugh (1986). Return predictability in the hedge fund industry has been investigated by Amenc et al. (2003), Hamza et al. (2006) and Vrontos (2009) and, more recently, in Wegener et al. (2010), Avramov et al. (2011) and Bali et al. (2011), among others. In particular, Wegener et al. (2010) take non-normality, heteroskedasticity and time-varying risk exposures into account to predict the conditional mean of the excess returns on four hedge fund strategies. With the same aim, Bali et al. (2011) exploit the hedge funds' exposures to various financial and macroeconomic risk factors. Avramov et al. (2011) find that macroeconomic variables, specifically the default spread and the Chicago Board Options Exchange volatility index (VIX), substantially improve the predictive ability of the benchmark linear pricing models used in the hedge fund industry. All these semi- 
nal papers are concerned with forecasting the expected excess returns but hardly pay any attention to higher moments of the conditional distribution that are relevant for investment decisions. This area of research has recently raised interest in the empirical finance literature on density forecasting, see Cenesiglou and Timmerman (2008) and Geweke and Amisano (2010), amongst others.

Efficient investment portfolios are usually the result of an optimization problem subject to some constraints. Thus, optimal portfolios are those that are in the mean-risk efficient frontier given some risk measure (e.g. variance) or are defined by the combination of risky and riskless assets that maximize a certain expected utility function representing investors' preferences. A powerful statistical method to solve both problems to comparing relative efficiency between investment portfolios is stochastic dominance tests. Fishburn (1977) shows that portfolios that are mean-risk efficient are also stochastically efficient and, hence, a portfolio that stochastically dominates another portfolio is also a better strategy in the mean-risk space. Similarly, this author shows that stochastic dominance implies an ordering of portfolios in terms of investors' expected utility maximization for general forms of the utility function and risk aversion levels.

Recent papers investigating investor behaviour report evidence of the importance of investment styles. According to the style investing hypothesis (Barberis and Shleifer, 2003), investors categorize risky assets into styles and subsequently allocate money to those styles depending on their relative performance. Hedge funds, like many other investment classes, are classified by investment styles. Ter Horst and Salganik (2011) find that better performing and more popular styles are rewarded with higher inflows in subsequent periods. Hence, the importance of predicting the performance of hedge fund investment styles.

The objective of this paper is to predict the relative performance one period ahead of hedge fund investment styles. We do this by means of dynamic stochastic dominance tests conditional on a time-varying information set. To forecast the conditional density corresponding to each hedge fund investment style, we propose nonparametric kernel methods. The vector of optimal bandwidth parameters is obtained as the solution of the cross- 
validation method introduced by Hall et al. (2004). This method automatically discards factors with no predictive power to forecast the return on the hedge fund style and, hence, provides very valuable information on the relevant set of predictive factors.

Our empirical application focuses on hedge fund investment styles that bet on financial markets movements. These investment styles fall into the broader category of 'Directional Traders', see Agarwal et al. (2004). Our sample period runs between 1994:01 and 2009:12, covering the recent global financial crisis in which these investment vehicles were more exposed to the ups and downs of financial markets than market-neutral strategies. In particular, we study the Dedicated Short Bias (DSB) style, that exhibits exposure to short positions, the Emerging Markets (EM) style, that focuses on investing in the securities of companies from emerging or developing countries, the Global Macro (GM) style, where bets are made on the direction of currency exchange rates or interest rates, and the Managed Futures (MF) style that exploits short-term patterns in futures markets. The predictive performance of these styles will also be compared to an asset-weighted portfolio, comprising the whole hedge fund industry, that we will call ALL. Our tests predict a superior performance of the GM investment style compared to the other styles under study. The DSB investment style is, on the other hand, stochastically dominated by the other directional styles. We also find that, whereas the DSB, EM and MF styles do not dominate or are dominated by ALL in the first order, indicating the relative efficiency of these strategies, for second order, we observe that ALL stochastically dominates these directional styles. This can be interpreted as a preference by risk-averse investors for exposure to the whole hedge fund industry compared to the directional styles. These results are confirmed by simple nonparametric tests on the difference of the observed realized excess returns.

The present study is also related to Li and Kazemi (2007), who estimated conditional density functions for hedge fund indices, and Meligkotsidou et al. (2009), who analyzed hedge fund investment styles using quantile regression methods. Our work is also connected with Billio et al. (2009), who studied hedge fund returns using nonparametric methods, and Giannikis and Vrontos (2011), who dealt with the non-linear relationship between 
hedge fund returns and risk factors using Bayesian model selection techniques and threshold models. Finally, we join Wong et al. (2008) and Li and Linton (2010) in applying stochastic dominance techniques to study the performance of hedge fund portfolios.

This article is structured as follows. Section 2 presents the nonparametric techniques used to predict the conditional density of returns of the different hedge fund styles and introduces the relevant dynamic tests of stochastic dominance between investment portfolios. Section 3 discusses the data analyzed and the results from the empirical application to the 'Directional Traders' hedge fund styles. Section 4 concludes. Tables and Figures are collected in an appendix.

\section{Methodology}

In this section, we first present the nonparametric kernel method to construct the predictive conditional density function. Second, we discuss dynamic stochastic dominance tests of arbitrary order.

\subsection{A Nonparametric Estimator for the Predictive Conditional Density}

Let $\left(Y_{t}\right)_{t \in \mathbf{Z}}$ be a strictly stationary time series process defined on a compact set $\Omega$, with an unconditional density function $f(y)$ and a cumulative distribution function $(c d f) F(y)$; let $f_{t-1}(y)$ and $F_{t-1}(y)$ be the corresponding predictive density and predictive distribution functions conditional on the sigma-algebra $\Im_{t-1}$ defined by all the information available up to time $t$. Our interest is in forecasting these functions. To do this, we consider a $k$-vector of predictive factors, denoted $X_{t}$, and a finite information set $I_{t}=\left\{\left(Y_{s}, X_{s}\right), t-m+1 \leq s \leq t\right\}$ defined on a compact set $\Omega^{\prime} \in \mathbf{R}^{q}$, with $q=(k+1) m$. With this set, we construct the predictive density function $f_{I_{t-1}}(y)$ that approximates $f_{t-1}(y)$. For completeness, we also introduce the multivariate density function of $I_{t}$, denoted $f^{I_{t}}(y)$, and its distributional counterpart, $F^{I_{t}}(y)$.

A natural nonparametric estimator of this conditional density for $I_{t-1}=x$, with $x$ being 
a multivariate vector that represents a realization of the recent history of the information set, is

$$
\widehat{f}_{x}(y)=\frac{n^{-1} \sum_{t=1}^{n} k_{h_{Y}}(y) W_{h}\left(I_{t-1}, x\right)}{\widehat{f}^{I_{1}}(x)},
$$

where $W_{h}\left(I_{t-1}, x\right)=\prod_{s=1}^{q} h_{s}^{-1} w\left(\frac{I_{t-1, s}-x_{s}}{h_{s}}\right)$, and $w(\cdot)$ and $k_{h_{Y}}(\cdot)$ are univariate kernel functions for the marginal random variables of the vectors $I_{t-1}$ and $Y_{t}$, respectively. The corresponding bandwidth parameters are $h_{s}, 1 \leq s \leq q$ and $h_{Y}$. The nonparametric estimator of $f^{I_{1}}(x)$ is $\widehat{f}^{I_{1}}(x)=n^{-1} \sum_{t=1}^{n} W_{h}\left(I_{t-1}, x\right) ; I_{t-1, s}$ and $x_{s}$ denote the $s^{t h}$-component of the multivariate random vectors $I_{t-1}$ and $x$, respectively. Li and Racine (2007) discuss the conditions for the uniform consistency of $(1)$ to $f_{x}(y)$ for all $(x, y) \in \Omega$.

In both theoretical and practical settings, nonparametric kernel estimation has been established as relatively insensitive to the choice of the kernel function. The same cannot be said for bandwidth selection, even more so in our setting given by the search for an appropriate information set $I_{t-1}$ to approximate $f_{t-1}(\cdot)$. Following Hall et al. (2004), we propose a maximum likelihood cross-validation method to determine the optimal vector of bandwidth parameters. This method allows us to empirically determine $I_{t-1}$, that is, the vector of conditioning variables that best predicts the density $f_{t-1}(y)$. The crossvalidation method automatically determines the irrelevant components of $\Im_{t-1}$ through assigning large smoothing parameters to them and, consequently, shrinking them toward the uniform distribution. The relevant components are precisely those that cross validation has chosen to smooth in a traditional way by assigning them bandwidth parameters of conventional size. A very nice review of the method and properties is given in $\mathrm{Li}$ and Racine (2007, Section 5.3).

The choice of the appropriate conditioning information set is very important to be able to optimally predict the density of returns and to implement the stochastic dominance tests. Note that one also needs to determine the forecasting scheme: fixed, rolling or recursive. To compare the predictive ability between density forecast competitors, we apply the test developed in Amisano and Giacomini (2007). This method assumes no knowledge of the 
true predictive density function and simply compares weighted versions of the predictive log-likelihood function of pairwise density forecast competitors over an out-of-sample period.

Let $\hat{f}_{x}(\cdot)$ and $\hat{g}_{x}(\cdot)$ be two competing forecasts of $f_{t-1}(y)$ at time $t-1$. The hypothesis test of relative predictive ability is the following;

$$
\begin{gathered}
H_{0}: E\left[W L R_{R, t+1}\right]=0, \quad t=1,2, \ldots, T \text { against } \\
H_{A}: E\left[\overline{W L R}_{R, P}\right] \neq 0 \text { for all } P \text { sufficiently large, }
\end{gathered}
$$

with $W L R_{R, t+1}=w\left(Y_{t+1}^{s t}\right)\left(\log \hat{f}_{x}\left(Y_{t+1}\right)-\log \hat{g}_{x}\left(Y_{t+1}\right)\right)$ and $\overline{W L R}_{R, P}=P^{-1} \sum_{t=R}^{T-1} W L R_{R, t+1}$; $Y_{t+1}^{s t}=\left(Y_{t+1}-\hat{\mu}_{R, t}\right) / \hat{\sigma}_{R, t}$ is the realization of the variable at time $t+1$, standardized using estimates of the unconditional mean and standard deviation of $Y_{t}, \hat{\mu}_{R, t}, \hat{\sigma}_{R, t}$, computed on the same sample on which the density forecasts are estimated. $R$ corresponds to the insample period and $P=T-R$ to the out-of-sample period. The weight function $w\left(Y_{t+1}^{s t}\right)$ can be arbitrarily chosen by the forecaster to select the desired region of the distribution of $Y_{t}$. The only requirement imposed on the weight function is that it be positive and bounded. Amisano and Giacomini (2007) propose different alternatives for the centre and the tails of the distribution of the random variable, that will be used in our empirical application.

The relevant test statistic for testing $H_{0}$ is

$$
t_{R, P}=\frac{\overline{W L R}_{R, P}}{\hat{\sigma}_{P} / \sqrt{P}}
$$

where $\hat{\sigma}_{P}$ is a heteroscedastic and autocorrelation consistent (HAC) estimator of the asymptotic long-run variance $\sigma_{P}^{2}=V\left(\sqrt{P} \overline{W L R}_{R, P}\right)$. At a significance level $\alpha$, this test rejects the null hypothesis of equal performance of forecasts whenever $\left|t_{R, P}\right|>z_{\alpha / 2}$, where $z_{\alpha / 2}$ is the $(1-\alpha / 2)$ quantile of a standard normal distribution. In the case of rejection, we would choose $f_{x}(\cdot)$ if $t_{R, P}$ is positive and $g_{x}(\cdot)$, otherwise.

We should highlight that this method and more recent improvements (Diks et al., 2011; Gneiting and Ranjan, 2011) do not allow us to determine the correct predictive specification 
of the model but they do permit the discrimination between potential forecasting methods. We will use these tests to choose between the fixed and rolling forecasting schemes in an out-of-sample evaluation. The definition of $I_{t}$ precludes the recursive forecasting scheme in our predictive exercise. Amisano and Giacomini (2007) also discard this method when implementing their predictive ability test for similar reasons.

\subsection{Dynamic Stochastic Dominance Tests}

Stochastic dominance provides a powerful methodology to compare investment styles. First order stochastic dominance compares the distribution function of returns; the second order compares the expected value of the distributions, and so on. An interesting interpretation of these measures is in terms of expected utilities for different degrees of investors' risk aversion. Thus, first order stochastic dominance implies the superiority of an investment strategy for risk-neutral investors. Similarly, second order implies the superiority of the strategy for risk-averse investors, and so on for higher orders of stochastic dominance and risk aversion. Seminal contributions to the topic are Stone (1973), Porter (1974) and Fishburn (1977).

In contrast to most of the literature on stochastic dominance, see Klecan et al. (1991), Anderson (1996), Davidson and Duclos (2000) and, more recently, Barrett and Donald (2003), we focus on a dynamic setting characterized by a time-varying information set recently proposed by Gonzalo and Olmo (2011). Similar tests, but with a different focus, are Linton et al. (2005), who test for stochastic dominance between residuals of parametric regression models, and Scaillet and Topaloglou (2010), who study stochastic dominance between stationary processes exhibiting weak dependence. Our approach is genuinely nonparametric and relies on the forecasts of the density functions discussed above.

Let A and B denote two investment portfolios; we say that A stochastically dominates B for order $\gamma$ conditional on the dynamic information set $I_{t-1}$ if, and only if,

$$
\Psi_{I_{t-1}, \gamma}^{A}(y) \leq \Psi_{I_{t-1}, \gamma}^{B}(y) \text { for all } y \in \Omega \text { and } t \in \mathbf{Z}
$$


with $\Psi_{I_{t-1}, \gamma}(y)=\int_{-\infty}^{y} \Psi_{I_{t-1}, \gamma-1}(\tau) d \tau$ and where $\Psi_{I_{t-1}, 1}(y)=F_{I_{t-1}}(y)$. The integration by parts of these quantities yields the following characterization of the stochastic dominance condition:

$$
\int_{-\infty}^{y}(y-\tau)^{\gamma-1} f_{I_{t-1}}^{A}(\tau) d \tau \leq \int_{-\infty}^{y}(y-\tau)^{\gamma-1} f_{I_{t-1}}^{B}(\tau) d \tau \text { for all } y \in \Omega \subset \mathbf{R} \text { and } t \in \mathbf{Z}
$$

We are interested in predicting the dynamics of the stochastic dominance relationship between investment styles. Our aim is to assess this condition for each period $t$. This implies that the conditioning information set $I_{t-1}$ for each $t$ is simply a vector $x$ describing the realization of the variable $I_{t-1}$. For simplicity in the exposition, we hereafter consider that $I_{t-1}=I_{t-1}^{A} \cup I_{t-1}^{B}$ refers to the set that collects the information contained in $I_{t-1}^{A}$ and $I_{t-1}^{B}$, with each of the latter sets containing the information relevant for forecasting $f_{t-1}^{A}$ and $f_{t-1}^{B}$, respectively.

Let $d_{t, \gamma}(y)=\left(y-Y_{t}^{A}\right)^{\gamma-1} 1\left(Y_{t}^{A} \leq y\right)-\left(y-Y_{t}^{B}\right)^{\gamma-1} 1\left(Y_{t}^{B} \leq y\right)$. Condition (6) characterizing predictive stochastic dominance of arbitrary order $\gamma \geq 1$ at time $t$ can be expressed, after some algebra, as the following composite hypothesis test:

$$
H_{0, \gamma}: E\left[d_{t, \gamma}(y) \mid I_{t-1}=x\right] \leq 0
$$

for all $y \in \Omega$ given $x$, against

$$
H_{1, \gamma}: E\left[d_{t, \gamma}(y) \mid I_{t-1}=x\right]>0
$$

for some $y \in \Omega$.

We follow the extant literature on stochastic dominance tests and focus on the subset of $H_{0, \gamma}$ given by the least favourable case and defined by the equality in $H_{0, \gamma}$ for all $y \in \Omega$; this test is denoted $\widetilde{H}_{0, \gamma}$. To test this condition, Gonzalo and Olmo (2011) propose the 
supremum of the following process on $y \in \Omega$,

$$
\widehat{D}_{\gamma}(y)=\frac{n^{-1} \sum_{t=1}^{n} d_{t, \gamma}(y) W_{h}\left(I_{t-1}, x\right)}{\widehat{f}^{I_{1}}(x)} .
$$

For $\gamma=1$ this expression is the difference between the nonparametric kernel estimators of the predictive distribution functions for the returns on portfolio A and $\mathrm{B}$, see $\mathrm{Li}$ and Racine (2007, p. 182). For $\gamma>1$, this expression compares higher moments of the conditional distribution of both portfolios.

Under $\widetilde{H}_{0, \gamma}$, Gonzalo and Olmo (2011) show that $T_{n, \gamma}=\left(n h_{1} \cdots h_{q}\right)^{1 / 2} \sup _{y \in \Omega} \widehat{D}_{\gamma}(y)$ converges in distribution to the supremum of a Gaussian process with zero mean and a covariance function that depends on the information set $I_{t-1}$, that is, on the vector $x$. For this reason, the asymptotic distribution of the test cannot be tabulated. Nevertheless, resampling and simulation methods can be implemented to approximate the asymptotic p-value of the tests. The following algorithm describes the procedure to approximate the p-value of the tests for a fixed period $t \in \mathbf{Z}$;

\section{Algorithm:}

1. Construct a grid of $m$ points $y_{1}, \ldots, y_{m}$ contained in the compact space $\Omega$ and execute the following steps for $j=1, \ldots, J$.

2. Generate $\left\{v_{t}\right\}_{t=1}^{n}$ independently and identically distributed (iid) $N(0,1)$ random variables.

3. Let $d_{t, \gamma}\left(y_{i}\right)=\left(y_{i}-Y_{t}^{A}\right)^{\gamma-1} 1\left(Y_{t}^{A} \leq y_{i}\right)-\left(y_{i}-Y_{t}^{B}\right)^{\gamma-1} 1\left(Y_{t}^{B} \leq y_{i}\right)$.

4. Set $\widehat{D}_{\gamma}^{*}\left(y_{i}\right)=\frac{n^{-1} \sum_{t=1}^{n} d_{t, \gamma}\left(y_{i}\right) W_{h}\left(I_{t-1}, x\right) v_{t}}{\widehat{f}^{I}(x)}$, with $x$ being a realization of $I_{t-1}$ and $h=$ $\left(h_{1}, \ldots, h_{q}\right)$ obtained from maximum likelihood cross-validation.

5. Compute $T_{n, \gamma}^{*(j)}=\left(n h_{1} \ldots h_{q}\right)^{1 / 2} \sup _{y_{i} \in \Omega} \widehat{D}_{\gamma}^{*}\left(y_{i}\right)$ for all $y_{i} \in \Omega$. 
This algorithm yields a random sample of $J$ observations from the distribution of the test statistic $T_{n, \gamma}$. The simulated p-value of the stochastic dominance test for a given order $\gamma$ is

$$
\widehat{p}_{n, \gamma}^{*}=\frac{1}{J} \sum_{j=1}^{J} 1\left(T_{n, \gamma}^{*(j)}>T_{n, \gamma}\right)
$$

which, under standard regularity conditions, see Hansen (1996) and Gonzalo and Olmo (2011), converges in probability to the true asymptotic p-value of $\widetilde{H}_{0, \gamma}$ as $J, n \rightarrow \infty$.

By repeating the test for each $t$ we can establish a time-varying ranking of portfolios that allows us to construct dynamic trading strategies based on conditional stochastic efficiency. This idea can be extended to analyze portfolio stochastic dominance/efficiency between more than two portfolios by using the test statistics proposed in Barrett and Donald (2003) and Linton et al. (2005). For illustrative purposes, we focus on pairwise comparisons in the next section.

\section{Empirical Application}

This section is divided into three blocks. The first part discusses the dataset given by the excess returns on four indices for the hedge fund investment styles included in the broad category 'Directional Traders' that bet on the direction of price movements of currencies, commodities, equities and bonds in the spot and futures markets. We also use the eight risk factors proposed by Fung and Hsieh that extend previous formulations. By means of statistical t-tests and the cross-validation bandwidth selection method, the second block derives the optimal set of factors for each investment style. This is done in a descriptive setting that extends standard linear pricing models for explaining the risk premium on hedge funds and in a predictive setting devised to optimally forecast the conditional density of returns. The final block of this section builds on this analysis to forecast the performance of the different hedge fund investment styles using the dynamic tests of stochastic dominance of orders one and two above. 


\subsection{Data description}

The hedge fund returns analyzed have been calculated from the Credit Suisse/Tremont asset-weighted indices expressed in US Dollars. ${ }^{1}$ Data have a monthly frequency and span the period 1994:01-2009:12. The investment styles considered are Dedicated Short Bias (DSB), Emerging Markets (EM), Global Macro (GM) and Managed Futures (MF). For completeness, we also study an asset-weighted portfolio comprising the whole hedge fund industry (ALL). In what follows, we will refer to the returns in excess over the risk-free asset (3-month Treasury Bill). For simplicity in the implementation of stochastic dominance tests, the returns are defined as the differences between the logarithm prices and not in percentage terms. Some descriptive statistics are reported in Table 1.

[Insert Table 1 about here]

On average, only GM obtains higher excess returns than ALL. The case of DSB, which obtains a negative mean return over the whole sample, is particularly relevant. In contrast, this style obtains the highest maximum return, while EM obtains the lowest minimum one. In terms of skewness, EM, GM and ALL display negative values, more pronounced for EM. For DSB, however, the positive skewness reveals the existence of very large positive returns. In addition, all hedge fund returns have excess kurtosis with the exception of MF, for which the Jarque-Bera test is not able to reject the null hypothesis of normality. Also in line with previously established evidence, the autocorrelation coefficients and the Ljung-Box statistic p-values suggest that the returns for DSB, GM and MF are serially correlated.

The set of explanatory factors for describing the hedge fund excess returns consists of the seven-factor model originally proposed by Fung and Hsieh (2004), which has been shown to achieve considerable explanatory power, plus an eighth factor recently proposed by these authors and given by the MSCI Emerging Market index monthly total excess return (MSCIEM). The seven-factor model includes three trend-following risk factors that are the

\footnotetext{
${ }^{1}$ http://www.hedgeindex.com
} 
excess returns on portfolios of lookback straddle options on bonds (BTF), currencies (CTF) and commodities (CMTF), constructed to replicate the maximum possible return to trendfollowing strategies on their respective underlying assets. ${ }^{2}$ The two equity-oriented risk factors are the excess monthly total return of the S\&P 500 index (EqMkt) and the Russell 2000 index monthly total return minus the S\&P 500 monthly total return (SizeSpr). Two bond-oriented factors are the monthly change in the 10-year Treasury constant maturity yield (BMkt) and the monthly change in the Moody's Baa yield minus the 10-year Treasury constant maturity yield (CrdSpr). The corresponding descriptive statistics for these factors are shown in Table 2.

[Insert Table 2 about here]

The Jarque-Bera test shows the non-normality of the risk factors. EqMkt, CrdSpr and MSCIEM display negative skewness and all risk factors have excess kurtosis; SizeSpr, CrdSpr and MSCIEM are serially correlated.

\subsection{Optimal Descriptive and Predictive Risk Factors}

Hall et al. (2004) show that cross-validation methods not only assign optimal weights to the different relevant factors for estimating a conditional density, but also automatically determine the factors that are irrelevant. Our interest in this nonparametric estimation procedure is twofold. First, from a descriptive perspective, we use this method to determine the risk factors with power to explain the excess returns observed in hedge funds; and second, from a forecasting perspective, we need to know the set of relevant factors for predicting the conditional density of hedge fund returns. In contrast to the standard linear pricing models popularized by Fung and Hsieh and other authors and to nonlinear refinements, we are interested in finding out which factors have power not only for describing (and predicting) the expected excess return but its complete density.

\footnotetext{
${ }^{2}$ Downloadable from http://faculty.fuqua.duke.edu/ dah7/HFRFData.htm.
} 
We consider three different specifications of the excess return both from a descriptive and a predictive point of view. For the former approach, the returns on the hedge fund are regressed on a set of factors measured on the same date and, for the latter, the set of factors is considered one period lagged. We use a simple linear regression model estimated by ordinary least squares (OLS), a quantile regression model for the $25^{t h}, 50^{\text {th }}$ and $75^{\text {th }}$ quantiles (QR25, QR50 and QR75, respectively), and the nonparametric conditional density estimation methods (NP). Our analysis covers the sample 1994:01-2009:12. The results of the descriptive approach are reported in Table 3.

[Insert Table 3 about here]

For the parametric methods, the risk factors are those variables that are found to be statistically significant at the $5 \%$ level; for the nonparametric alternative, the relevant factors are those for which the cross-validation bandwidth selection rule assigns a value lower than one. Our findings can be summarized as follows. First, the set of significant risk factors depends on the investment style. The Fung-Hsieh linear pricing model reveals that the equity-oriented risk factors (EqMkt and SizeSpr) are sufficient to explain the risk premium of the DSB style. For the EM style, the relevant risk factor is the emerging market index (MSCIEM) and the pricing model resembles a standard CAPM. Second, for a given style, the relevant risk factors depend on the statistical measure under scrutiny. For example, the number of relevant factors for the GM style decreases as we move towards the upper region of the distribution. It is also interesting to observe that, for this style, the equity-oriented factors lose explanatory power beyond the lower tail of the return distribution. The assetweighted portfolio comprising the whole hedge fund industry (ALL) is explained by the largest number of factors across different statistical measures. Third, the analysis of the whole conditional distribution through the nonparametric approach considers the largest set of explanatory factors. For example, seven out of eight potential factors are considered for explaining the conditional density of the MF style returns. Finally, and unsurprisingly, the nonparametric method achieves the highest log-likelihood values. 
The above results dramatically change in the predictive framework. Following Wegener et al. (2010), we also include the lagged hedge fund excess return as a potential predictive factor. Results for the overall sample are reported in Table 4. The standard linear pricing model lacks any predictive power for the directional styles, except for GM. In addition, the risk factors are very poor in predicting the quantiles of the hedge fund returns distribution. Nonetheless, bond related factors (BTF, BMkt and CrdSpr) have some predictive power for GM and, especially, for ALL. Analogous to the descriptive approach, the nonparametric method makes use of the largest number of risk factors for constructing the conditional predictive density. Interestingly, these factors are roughly the same as those of the descriptive exercise previously discussed. This exercise reveals the importance of obtaining nonparametric estimates of the conditional predictive density of returns. In contrast to standard linear pricing formulations, we have found that the Fung-Hsieh risk factors also have predictive power when properly exploited.

[Insert Table 4 about here]

To assess the persistence of these factors, we compare the forecasting ability of the rolling and fixed forecasting schemes. Whereas, in the former scheme, the bandwidth parameters are recomputed for each rolling window, in the fixed scheme, the bandwidth parameter vector for estimating the conditional predictive density remains constant. The evolution in the dynamics of the optimal bandwidth parameters obtained through the rolling scheme provide very valuable information on the ability of the factors to predict the conditional density over the out-of-sample period. If the optimal bandwidth parameter corresponding to a potential predictive factor is stable along the out-of-sample evaluation period and takes values of conventional size, there is evidence of the persistence of this factor for predicting the conditional density of returns. On the other hand, an erratic behaviour in the dynamics of the optimal bandwidth parameter is evidence of abrupt changes in the predictive ability of the factor. We implement the predictive ability test developed by Amisano and Giacomini 
(2007) between the density forecast obtained from (1) using a fixed forecasting scheme (the bandwidth parameters are obtained from the the period 1994:01-2006:12) and from a rolling forecasting scheme in which the bandwidth parameters are re-estimated for each one-monthahead rolling window. The results of the test in Table 5 reflect a strong persistence in the predictive ability of the factors revealed in Table 4 . There are no statistical differences in predictive ability between the fixed and rolling approaches. Figure 1 depicts the dynamics of the optimal bandwidth parameters for DSB and the factors BTF, EqMkt, SizeSpr and MSCIEM. The dynamics of these parameters are reasonably stable over the rolling out-ofsample evaluation period and give support to the results of the predictive ability test.

[Insert Figure 1 about here]

For completeness, Table 6 reports the set of fixed optimal bandwidth parameters corresponding to each potential predictive factor from our set of variables. The results highlight the differences between styles and factors. Unsurprisingly, the only factor with power for all styles is the lagged return; BTF and EQMkt also have an important weight across styles. CMTF, on the other hand, is only significant for the conditional density of ALL.

[Insert Tables 5 and 6 about here]

\subsection{Forecasting the Performance of Hedge Fund Styles}

The aim of this study is to predict, one period ahead, the best investment strategy from the set of hedge fund styles involved in directional trading. The null hypothesis of interest is $\widetilde{H}_{0, \gamma}: E\left[d_{t, \gamma}(y) \mid I_{t-1}=x\right]=0$ for all $y \in \Omega$ with $t$ fixed. The test is one-sided and has power against the hypothesis $E\left[d_{t, \gamma}(y) \mid I_{t-1}=x\right]>0$. This test is implemented for all $t$ in the out-of-sample evaluation period using a rolling scheme to incorporate the information into the test. Expression (9) is estimated using rolling windows of size $R=160$. For the 
first out-of-sample observation, we use the sample 1994:01-2006:12 to construct $\widehat{D}_{\gamma}(y)$ and simulate the p-value of the test. This exercise is repeated for 1994:02-2007:01 and so on to obtain a time series of 36 p-values over the period 2007:01-2009:12. The optimal bandwidth parameter vector corresponding to $I_{t-1}$ is a fixed vector $\left(h_{1, A}, \ldots, h_{q, A}, h_{1, B}, \ldots, h_{q^{\prime}, B}\right)$ with $q+q^{\prime}$ being the number of factors relevant for at least one of the strategies and that contains the cross-validation optimal bandwidth parameters corresponding to the conditional predictive density (1) for portfolios A and B. This vector can be constructed by composing the vectors for each strategy reported in Table 6. Other bandwidth parameter choices have also been implemented, e.g. $h_{i}=0.5$ (ad-hoc choice) and $h_{i}=n^{-1 /\left(4+\left(q+q^{\prime}\right)\right)}$ (optimal rate of convergence for the bandwidth) for $n=R=160$ and $i=1, \ldots, q+q^{\prime}$, yielding very similar results.

Figure 2 plots the dynamic p-values of stochastic dominance tests of order one for each directional investment style against ALL. The dashed line reflects the p-value of the test whose null hypothesis is given by the stochastic dominance of ALL over the individual styles. The solid line represents the p-value of the test defined by the converse null hypothesis, that is, the individual style dominates ALL for order one. Similarly, Figure 3 plots the dynamic p-values of the tests between all the possible pairwise combinations of directional investment styles. For DSB vs. EM, for example, the dashed line corresponds to the p-value of the test with null hypothesis given by the dominance of DSB over EM and the solid line reports the p-values of the converse hypothesis.

[Insert Figures 2 and 3 about here]

The test for first order stochastic dominance predicts the outperformance of GM over the other investment styles, ALL included, after the third quarter of 2007. The interpretation of this result is simple; the conditional predictive distribution of the GM returns is always to the left of those characterizing the other investment styles. Investors can use this information to decide, at time $t$, where to invest at time $t+1$. Obviously, the test indicates that GM is a superior investment strategy for both risk-neutral and risk-averse individuals. 
Pairwise comparisons between ALL and each of the DSB, EM and MF styles (Figure 2) reveal that these investment styles are stochastically efficient in the sense that no investment dominates the other in the first order. Following the characterization of risk aversion and the degrees of stochastic dominance given by Fishburn (1977), risk-neutral investors should be indifferent over the out-of-sample period between ALL and these different styles. The results of the test, in Figure 3, do provide more valuable information. Surprisingly, DSB is dominated during the crisis period by the other individual styles, questioning the value of this strategy compared, for example, to GM or EM; the test also predicts that EM dominates MF. Interestingly, the test of first order stochastic dominance predicts a very clear ranking of investment performance over the whole out-of-sample period. The dominant styles (GM and EM) share a strong exposure to global bond and equity factors, more importantly for GM. EM seems to be isolated from the other financial markets and to be driven by movements of equity markets in developing economies. The p-values of the tests for second order stochastic dominance are in Figures 4 and 5.

[Insert Figures 4 and 5 about here]

Fishburn (1977) shows that, if portfolio A dominates portfolio B in the first order, it also dominates it for higher orders of stochastic dominance. Our empirical findings are consistent with this theory. GM also dominates the other portfolios and DSB is dominated by the other directional traders styles. In contrast to the study of first order dominance, this test predicts that ALL is a superior investment to DSB, EM and MF for most of the outof-sample evaluation period. Using these results, we expect that risk-averse investors would bet on this diversified investment strategy rather than investing in any of the individual directional styles, with the exception of GM which exhibits substantial higher returns for lower levels of volatility.

It is worth noting that these results are predictions of future performance and, hence, in order to be accepted, they should be compared to ex-post performance. Following the 
related literature, we propose simple nonparametric proportion tests to assess the difference between the excess returns realized over the out-of-sample period. Let $r_{t}^{A}$ and $r_{t}^{B}$ denote these realized excess returns for two different investment strategies $A$ and $B$ and let $z_{t}=$ $r_{t}^{A}-r_{t}^{B}$. We say that strategy $A$ has been better than $B$ for risk-neutral investors if $p_{z}=P\left\{z_{t}>0\right\}>0.5$. Similarly, we say that strategy $A$ is better than $B$ for risk-averse investors if $\widetilde{p}_{z}=P\left\{\widetilde{z}_{t}>0\right\}>0.5$ with $\widetilde{z}_{t}=r_{t}^{A} / \sigma_{A}-r_{t}^{B} / \sigma_{B}$ where $\sigma_{A}$ and $\sigma_{B}$ are the unconditional standard deviations of the returns on $A$ and $B$ over the out-of-sample evaluation period. These conditions can be tested as follows ${ }^{3}$ :

$$
H_{0}^{(n)}: p_{z} \leq 0.5 \text { against } H_{1}^{(n)}: p_{z}>0.5
$$

To test this condition over an evaluation period of length $P$, we propose the sample version of $p_{z}$ given by $\widehat{p}_{z}=\frac{1}{P} \sum_{t=1}^{P} 1\left(z_{t}>0\right)$. If $z_{t}$ is serially uncorrelated, it is well known that the test statistic $\sqrt{P} \frac{\widehat{p}_{z}-p_{z}}{\sqrt{\widehat{p}_{z}\left(1-\widehat{p}_{z}\right)}}$ converges, as $P \rightarrow \infty$, to a standard normal distribution. Otherwise, we need to correct for the existence of serial correlation between the sequence of indicator functions. One possibility is to estimate the variance of $\widehat{p}_{z}$ using serially dependent robust estimators. These estimators provide a nice alternative for moderate sample sizes. For small values of $P$, block bootstrap methods are more suitable to approximate the distribution of the relevant test statistic defined now by $S_{P}=\sqrt{P}\left(\widehat{p}_{z}-p_{z}\right)$.

These resampling methods are based on blocking arguments, in which data are divided into blocks that are resampled. The artificial time series obtained from this resampling procedure are of the same size as the original sample and mimic the dependence structure observed in the data. Let $b, l$ denote integers such that $P=b l$, with $b$ the block size. There are two different ways of implementing block bootstrap depending on whether the blocks are overlapping or non-overlapping. The overlapping rule produces $P-l+1$ blocks of consecutive observations. We focus on the non-overlapping method that yields a sample of size $P$ from $l$ disjoint blocks $B_{1}, \ldots, B_{l}$ of size $b$, with $B_{j}=\left(1\left(z_{1+(j-1) b}>0\right), \ldots, 1\left(z_{j b}>0\right)\right)$ and $j=$

\footnotetext{
${ }^{3}$ The test for $\widetilde{p}_{z}$ is analogous and is omitted to save space.
} 
$1, \ldots, l$. As in the $i$ id bootstrap, the blocks can be repeated when resampling randomly with replacement. The asymptotic distribution of the out-of-sample test $S_{P}$ can be approximated by the empirical distribution of the test statistic sequence $S_{P, i}=\sqrt{P}\left(\widehat{p}_{z, i}^{*}-\widehat{p}_{z}\right)$, indexed by $i=1, \ldots, M$, with $M$ the number of Monte Carlo simulations, and $\widehat{p}_{z, i}^{*}$ the bootstrap counterpart of $\widehat{p}_{z}$ constructed from the simulated block-bootstrap sample $i$. The empirical p-value of the test is obtained as

$$
\widehat{p}_{P, b}=\frac{1}{M} \sum_{i=1}^{M} 1\left(S_{P, i}>S_{P}\right)
$$

[Insert Table 7 about here]

Table 7 reports the results of the test $H_{0}^{(n)}$ for $p_{z}$ and $\widetilde{p}_{z}$ equal to 0.5. Our choice of block size follows from the optimal data-driven algorithm of Politis et al. (2009). It varies across experiments with an average block size $(b)$ of 2.223 and a standard deviation of 0.677 . The number of Monte Carlo simulations is 2,000 . For both $p_{z}$ and $\widetilde{p}_{z}$ we observe that the GM style slightly outperforms the other investment styles ex-post, the test being statistically significant at the 5\% level against DSB and MF. In addition, the results for ALL confirm that this style has not dominated the other styles over the evaluation period as our tests for $\gamma=1$ suggested. However, for $\gamma=2$, only the prediction for DSB is in line with the empirical ex-post test. Finally, the findings for the DSB style reveal its ex-post underperformance against the other strategies.

\section{Concluding remarks}

The aim of this study is to predict, one period ahead, the best investment strategy from a set of potential candidates. This is done by constructing a predictive test of stochastic dominance of arbitrary order that is applied to directional hedge fund investment styles during the recent global financial crisis. The empirical results provide a clear answer to 
the question of which style to choose one period ahead. The tests of stochastic dominance of order one and two consistently predict that the Global Macro style is superior to the other investment alternatives under consideration: Dedicated Short Bias, Emerging Markets, Managed Futures and an asset-weighted portfolio comprising hedge funds from the whole industry. The very popular Dedicated Short Bias style is found to be dominated by the other styles. For risk-averse investors, the whole industry portfolio is an interesting option that dominates all directional styles except the Global Macro style in the second order.

A byproduct of our analysis has been the study of the optimal set of factors for describing as well as predicting the excess returns on hedge funds. The standard linear pricing formulation for modelling the risk premium on the returns has been extended to analyze the whole predictive density of returns. The cross-validation bandwidth selection method used for estimating these conditional density functions nonparametrically has been instrumental for determining which factors have potential for predicting the risk premium. For the linear pricing model, the factors proposed by Fung and Hsieh to explain the risk premium barely have predictive ability one period ahead. Interestingly, these factors are found to be highly significant if we consider the whole predictive density of returns instead. The nature of these factors depends on the style under consideration. 


\section{References}

[1] Agarwal, V., N. Daniel and N. Naik, 2004. Flows, performance, and managerial incentives in the hedge fund industry. Mimeo.

[2] Agarwal, V. and N. Naik, 2004. Risks and portfolio decisions involving hedge funds. Review of Financial Studies 17(1), 63-98.

[3] Amenc, N., S. El Bied and L. Martellini, 2003. Predictability in Hedge Fund Returns. Financial Analysts Journal 59(5), 32-46.

[4] Amenc, N. and L. Martinelli, 2002. Portfolio optimization and hedge fund style allocation decisions. Journal of Alternative Investments 5, 7-20.

[5] Amisano, G. and R. Giacomini, 2007. Comparing Density Forecasts via Weighted Likelihood Ratio Tests. Journal of Business and Economic Statistics 25(2), 177-190.

[6] Anderson, G, 1996. Nonparametric Tests of Stochastic Dominance in Income Distributions. Econometrica 64, 1183-1193.

[7] Avramov, D., R. Kosowski, N. Naik and M. Teo, 2011. Hedge funds, managerial skill, and macroeconomic variables. Journal of Financial Economics 99, 672-692.

[8] Bali, T., S. Brown and M. Caglayan, 2011. Do Hedge Funds' Exposures to Risk Factors Predict Their Future Returns? Journal of Financial Economics 101(1), 36-68.

[9] Barberis, N. and A. Shleifer, 2003. Style Investing. Journal of Financial Economics 68, 161-199.

[10] Barrett, G. and S. Donald, 2003. Consistent tests for stochastic dominance. Econometrica $71,71-104$.

[11] Billio, M., M. Getmansky and L. Pelizzon, 2009. Nonparametric Analysis of Hedge Fund Returns: New Insights from High Frequency Data. Journal of Alternative Investments 12(1), 21-38. 
[12] Cappoci, D. and G. Hübner, 2004. Analysis of hedge fund performance. Journal of Empirical Finance 11, 55-89.

[13] Cenesiglou, T. and A. Timmermann, 2008. Is the Distribution of Stock Returns Predictable? Mimeo.

[14] Davidson, R. and J. Duclos, 2000. Statistical inference for stochastic dominance and for the measurement of povery and inequality. Econometrica 68, 1435-1464.

[15] Diks, C., V. Panchenko and D. van Dijk, 2011. Likelihood-based scoring rules for comparing density forecasts in tails. Journal of Econometrics 163(2), 215-230.

[16] Eling, M. and R. Faust, 2010. The performance of hedge funds and mutual funds in emerging markets. Journal of Banking and Finance 34(8), 1993-2009.

[17] Fishburn, P., 1977. Mean-Risk Analysis with Risk Associated with Below-Target Returns. American Economic Review 67(2), 116-126.

[18] Fung, W. and D. Hsieh, 1997. Empirical Characteristics of Dynamic Trading Strategies: The Case of Hedge Funds. Review of Financial Studies 10, 275-302.

[19] Fung, W. and D. Hsieh, 2001. The risk in hedge fund strategies: Theory and evidence from trend followers. Review of Financial Studies 14, 313-341.

[20] Fung, W. and D. Hsieh, 2002. The risk in fixed-income hedge fund styles. Journal of Fixed Income 12, 6-27.

[21] Fung, W. and D. Hsieh, 2004a. Hedge fund benchmarks: A risk based approach. Financial Analysts Journal 60(5), 65-80.

[22] Fung, W. and D. Hsieh, 2004b. The risk in hedge fund strategies: Theory and evidence from long/short equity hedge funds. Mimeo.

[23] Geweke, J. and G. Amisano, 2010. Comparing and evaluating Bayesian predictive distributions of asset returns. International Journal of Forecasting 26, 216-230. 
[24] Giannikis, D. and I. Vrontos, 2011. A Bayesian approach to detecting nonlinear risk exposures in hedge fund strategies. Journal of Banking and Finance 35(6), 1399-1414.

[25] Gneiting, T. and R. Ranjan, 2011. Comparing density forecasts using threshold- and quantile-weighted scoring rules. Journal of Business and Economic Statistics 29(3), 411-422.

[26] Gonzalo, J. and J. Olmo, 2011. Conditional Stochastic Dominance Tests in Dynamic Settings. Mimeo.

[27] Hall, P., J. Racine and Q. Li, 2004. Cross-Validation and the Estimation of Conditional Probability Densities. Journal of the American Statistical Association 99, 1015-1026.

[28] Hamza, O., M. Kooli and M. Roberge, 2006. Further Evidence on Hedge Fund Return Predictability. The Journal of Wealth Management, 9(3), 68-79.

[29] Hansen, B., 1996. Inference when a nuisance parameter is not identified under the null hypothesis. Econometrica 64(2), 413-430.

[30] Keim, D. B. and R. F. Stambaugh, 1986. Predicting Returns in the Stock and Bond Markets. Journal of Financial Economics 17(2), 357-390.

[31] Klecan, L., R. McFadden and R. McFadden, 1991. A Robust Test for Stochastic Dominance. Mimeo.

[32] Li, Y. and H. Kazemi, 2007. Conditional Properties of Hedge Funds: Evidence from Daily Returns. European Financial Management 13(2), 211-238.

[33] Li, S. and O. Linton, 2010. Evaluating Hedge Fund Performance: A Stochastic Dominance Approach. In Handbook of Portfolio Construction: Contemporary Applications of Markowitz Techniques (J. B. Guerard, ed.), 551-564.

[34] Li, Q. and J. S. Racine, 2007. Nonparametric Econometrics. Princeton University Press. 
[35] Linton, O., E. Maasoumi and Y. Whang, 2005. Consistent testing for stochastic dominance under general sampling schemes. Review of Economic Studies 72, 735-765.

[36] Meligkotsidou, L., I. Vrontos and S. Vrontos, 2009. Quantile regression analysis of hedge fund strategies. Journal of Empirical Finance 16, 264-279.

[37] Patton, A., 2009. Are "Market Neutral" Hedge Funds Really Market Neutral? Review of Financial Studies 22(7), 2495-2530.

[38] Politis, D., H. White and A. Patton, 2009. Correction: Automatic Block-Length Selection for the Dependent Bootstrap. Econometric Reviews 28, 373-375.

[39] Porter, R.B., 1974. Semivariance and Stochastic Dominance: A Comparison. American Economic Review 64, 200-204.

[40] Scaillet, O. and N. Topaglou, 2010. Testing for Stochastic Dominance Efficiency. Journal of Business and Economic Statistics 28(1), 169-180.

[41] Stone, B.K., 1973. A General Class of Three-Parameter Risk Measures. Journal of Finance 28, 675-685.

[42] Ter Horst, J. and G. Salganik, 2011. Style Chasing by Hedge Fund Investors. Mimeo.

[43] Vrontos, I., 2009. Evidence for Hedge Fund Predictability from a Multivariate Student-t Full-Factor GARCH Model. Mimeo.

[44] Wegener, C., R. von Nitzsch and C. Cengiz, 2010. An advanced perspective on the predictability in hedge fund returns. Journal of Banking and Finance 34(11), 26942708 .

[45] Wong, W-K., K. Phoon and H. Lean, 2008. Stochastic dominance analysis of Asian hedge funds. Pacific-Basin Finance Journal 16, 204-223. 
Table 1. Descriptive statistics of excess returns. 'Directional Traders' hedge fund investment styles, 1994:01-2009:12.

\begin{tabular}{lccccc}
\hline \hline & ALL & DSB & EM & GM & MF \\
\hline Mean & 0.005 & -0.004 & 0.005 & 0.007 & 0.003 \\
Median & 0.005 & -0.008 & 0.011 & 0.008 & 0.0002 \\
Maximum & 0.081 & 0.223 & 0.160 & 0.101 & 0.095 \\
Minimum & -0.080 & -0.096 & -0.234 & -0.119 & -0.098 \\
Std. Dev. & 0.022 & 0.049 & 0.045 & 0.030 & 0.034 \\
Skewness & -0.268 & 0.737 & -0.799 & -0.101 & 0.033 \\
Kurtosis & 5.293 & 4.545 & 7.648 & 6.145 & 3.064 \\
Jarque-Bera & 44.384 & 36.459 & 193.241 & 79.457 & 0.069 \\
p-value & 0.000 & 0.000 & 0.000 & 0.000 & 0.966 \\
AC(1) & 0.202 & 0.093 & 0.320 & 0.084 & 0.069 \\
p-value & 0.005 & 0.194 & 0.000 & 0.240 & 0.333 \\
AC(4) & -0.037 & -0.060 & -0.032 & -0.072 & 0.004 \\
p-value & 0.036 & 0.424 & 0.000 & 0.465 & 0.122 \\
AC(12) & -0.010 & -0.134 & -0.041 & 0.007 & -0.057 \\
p-value & 0.132 & 0.437 & 0.002 & 0.031 & 0.022 \\
Observations & 192 & 192 & 192 & 192 & 192 \\
\hline
\end{tabular}

Note: DSB: Dedicated Short Bias, EM: Emerging Markets, GM: Global Macro, MF: Managed Futures. 
Table 2. Descriptive statistics of Fung-Hsieh hedge fund risk factors, 1994:01-2009:12.

\begin{tabular}{lcccccccc}
\hline \hline & BTF & CTF & CMTF & EqMkt & SizeSpr & BMkt & CrdSpr & MSCIEM \\
\hline Mean & -0.017 & -0.001 & -0.006 & 0.003 & 0.0008 & -0.003 & -0.0005 & 0.003 \\
Median & -0.051 & -0.045 & -0.032 & 0.009 & 0.0003 & -0.004 & 0.000 & 0.005 \\
Maximum & 0.684 & 0.898 & 0.644 & 0.100 & 0.184 & 0.275 & 0.216 & 0.166 \\
Minimum & -0.256 & -0.304 & -0.234 & -0.168 & -0.163 & -0.269 & -0.253 & -0.297 \\
Std. Dev. & 0.147 & 0.198 & 0.139 & 0.045 & 0.036 & 0.066 & 0.053 & 0.072 \\
Skewness & 1.459 & 1.366 & 1.263 & -0.712 & 0.282 & 0.469 & -0.491 & -0.776 \\
Kurtosis & 5.995 & 5.623 & 5.532 & 4.096 & 7.479 & 7.043 & 8.276 & 4.874 \\
Jarque-Bera & 139.846 & 114.796 & 102.347 & 25.821 & 163.069 & 137.833 & 230.410 & 47.378 \\
p-value & 0.000 & 0.000 & 0.000 & 0.000 & 0.000 & 0.000 & 0.000 & 0.000 \\
AC(1) & 0.119 & 0.035 & -0.038 & 0.112 & -0.136 & 0.037 & 0.199 & 0.211 \\
p-value & 0.096 & 0.629 & 0.595 & 0.117 & 0.058 & 0.610 & 0.005 & 0.003 \\
AC(4) & -0.060 & -0.084 & 0.005 & 0.066 & -0.035 & 0.009 & -0.001 & -0.026 \\
p-value & 0.435 & 0.115 & 0.988 & 0.254 & 0.286 & 0.001 & 0.012 & 0.017 \\
AC(12) & -0.018 & -0.074 & 0.027 & 0.053 & 0.039 & 0.008 & 0.079 & -0.027 \\
p-value & 0.406 & 0.445 & 0.991 & 0.614 & 0.307 & 0.009 & 0.002 & 0.090 \\
Observations & 192 & 192 & 192 & 192 & 192 & 192 & 192 & 192 \\
\hline
\end{tabular}

Note: BTF: Excess returns on portfolios of lookback straddle options on bonds, CTF: on currencies, CMTF: on commodities, EqMkt: Excess monthly total return of the S\&P500 index, SizeSpr: Russell 2000 index monthly total return minus the S\&P500 monthly total return, BMkt: Monthly change in the 10-year Treasury constant maturity yield, CrdSpr: Monthly change in the Moody's Baa yield minus the 10-year Treasury constant maturity yield, MSCIEM: MSCI Emerging Market index monthly total excess return. 


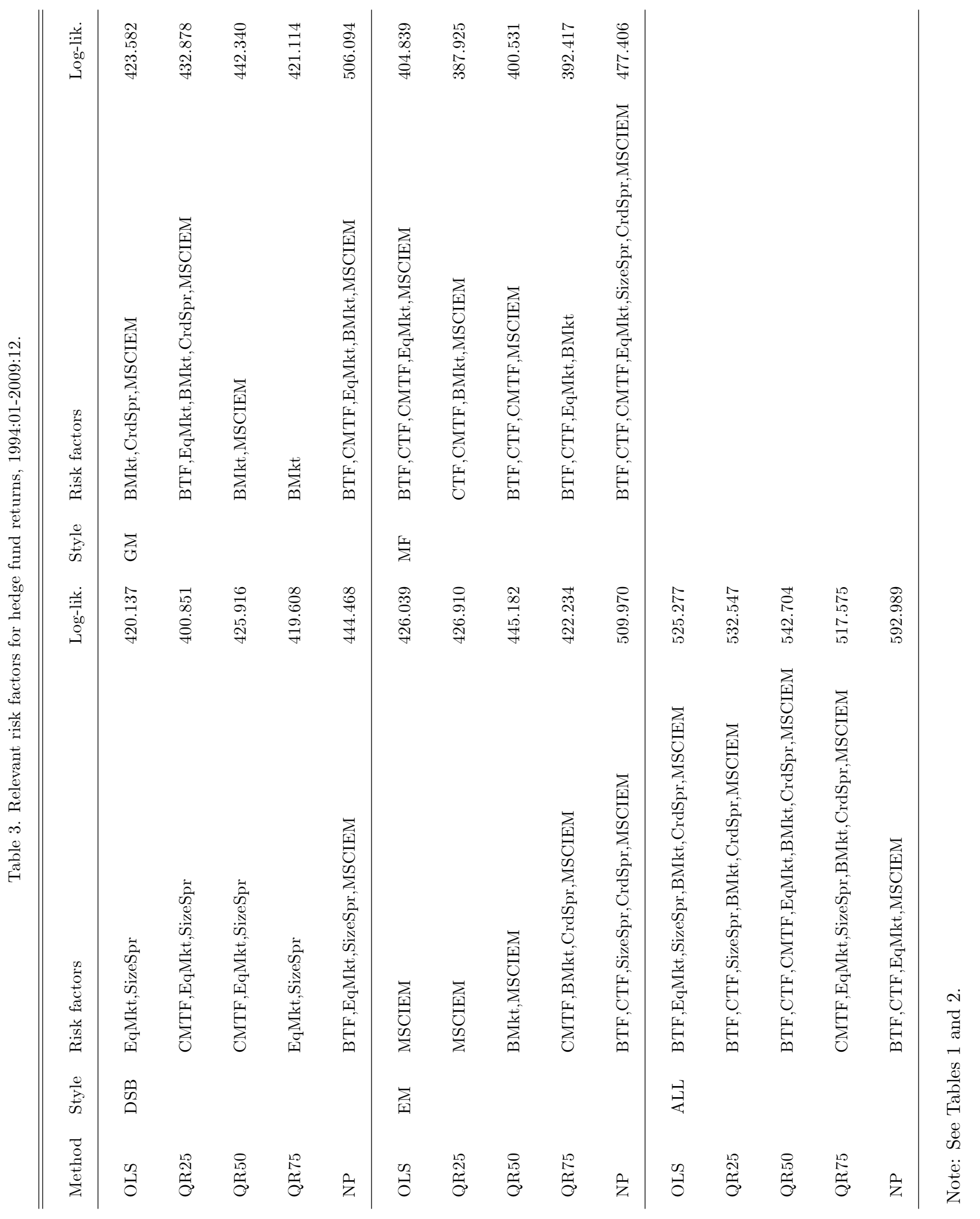




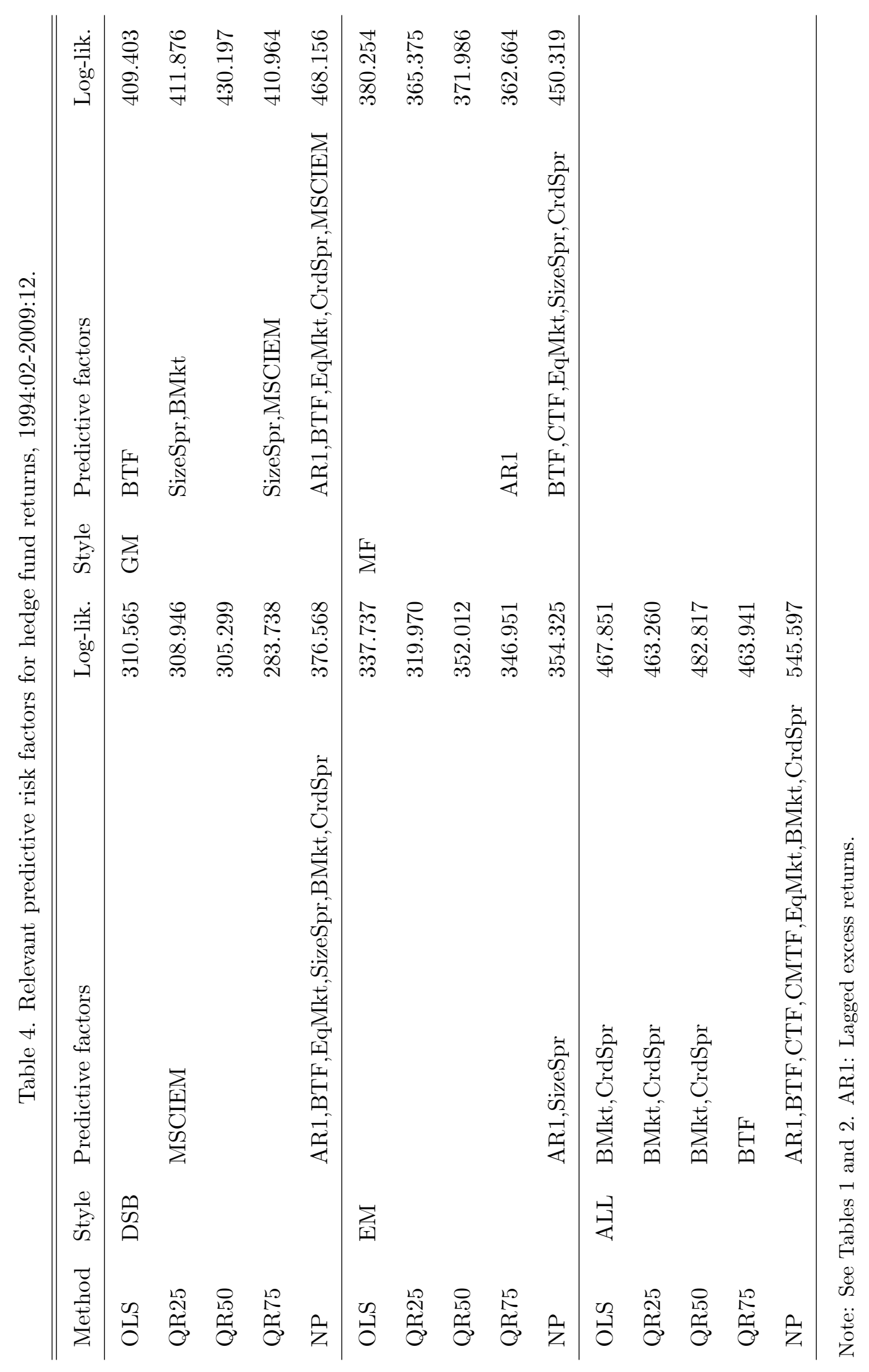



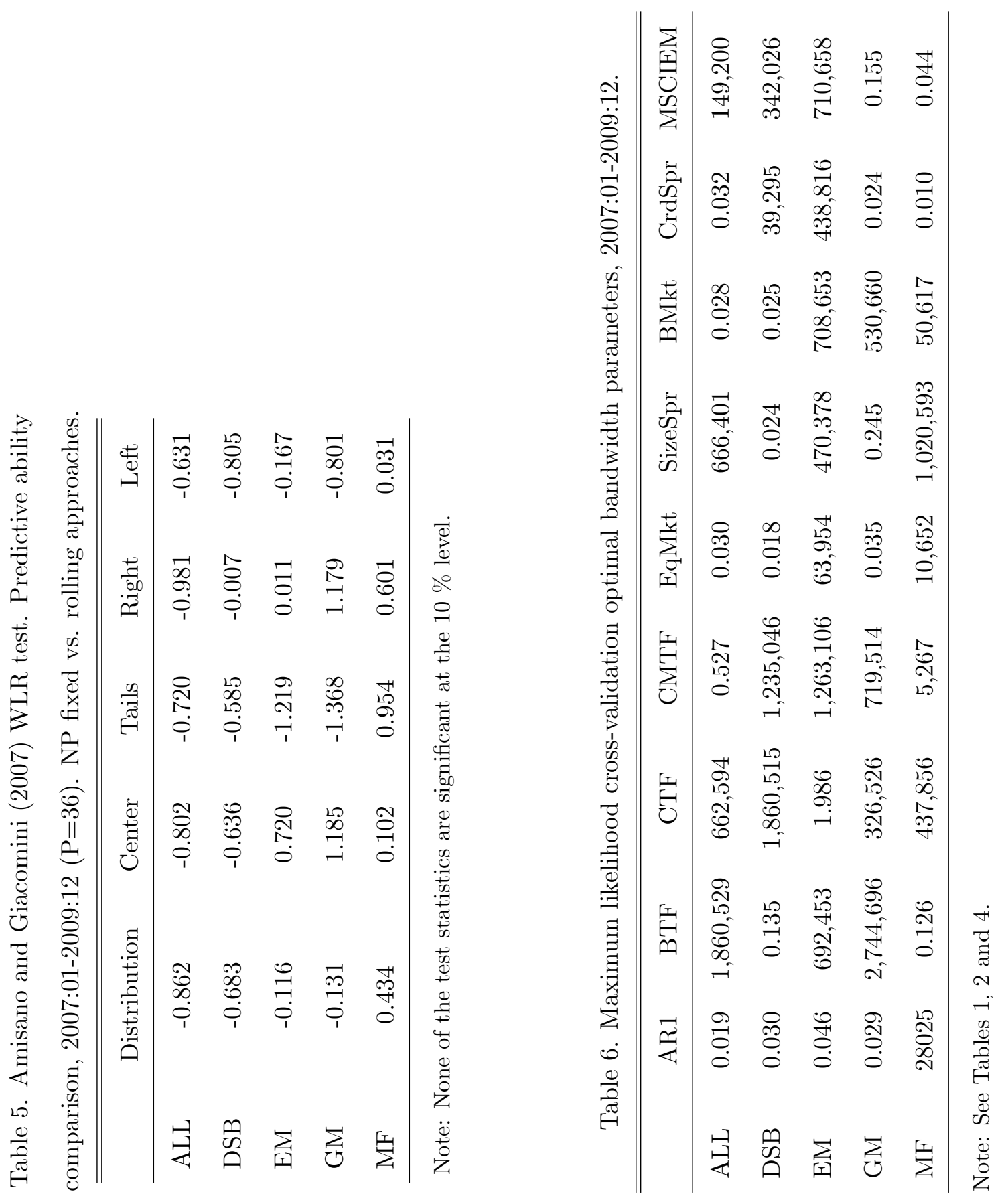


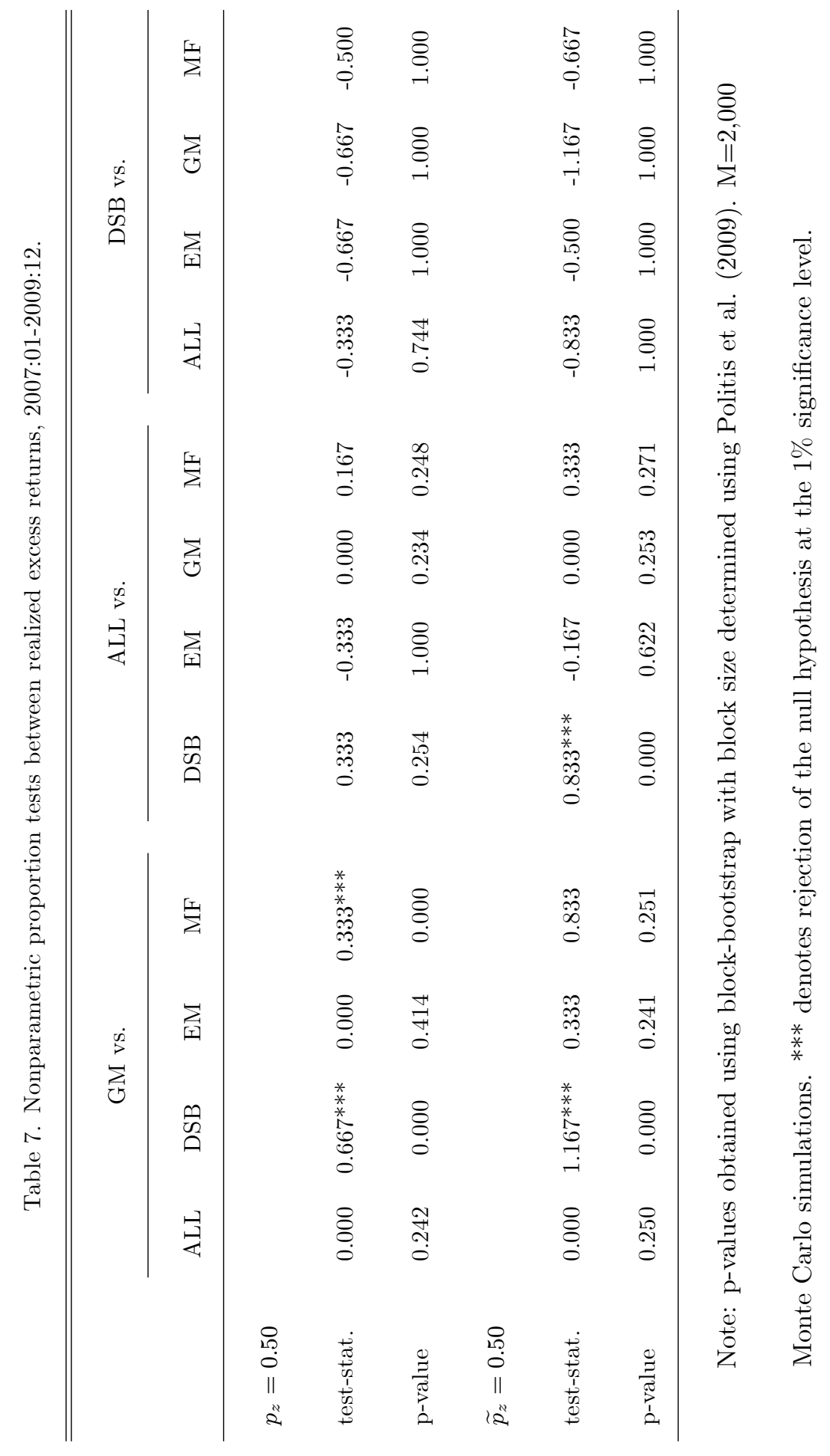




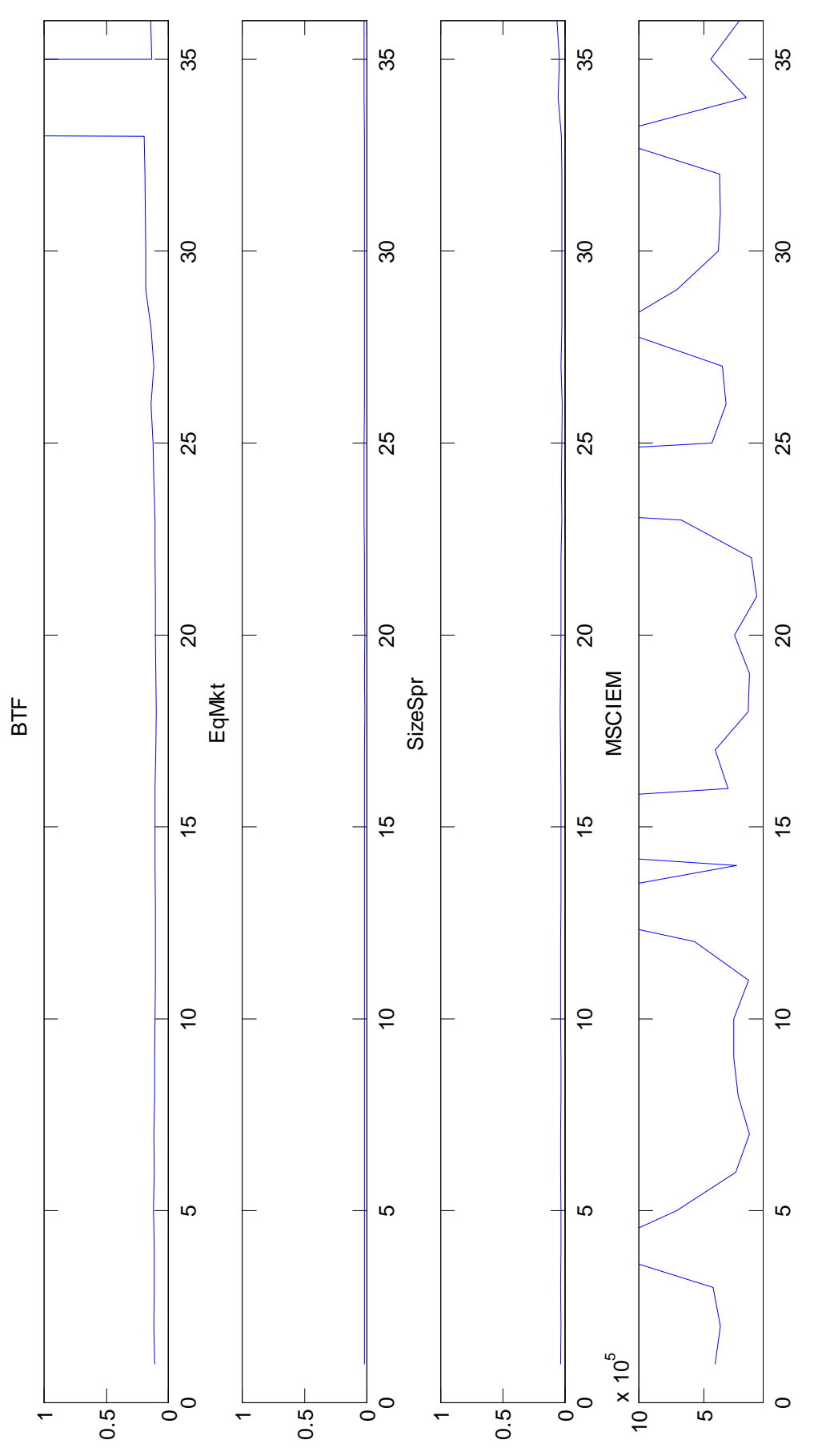

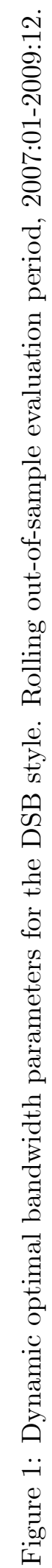



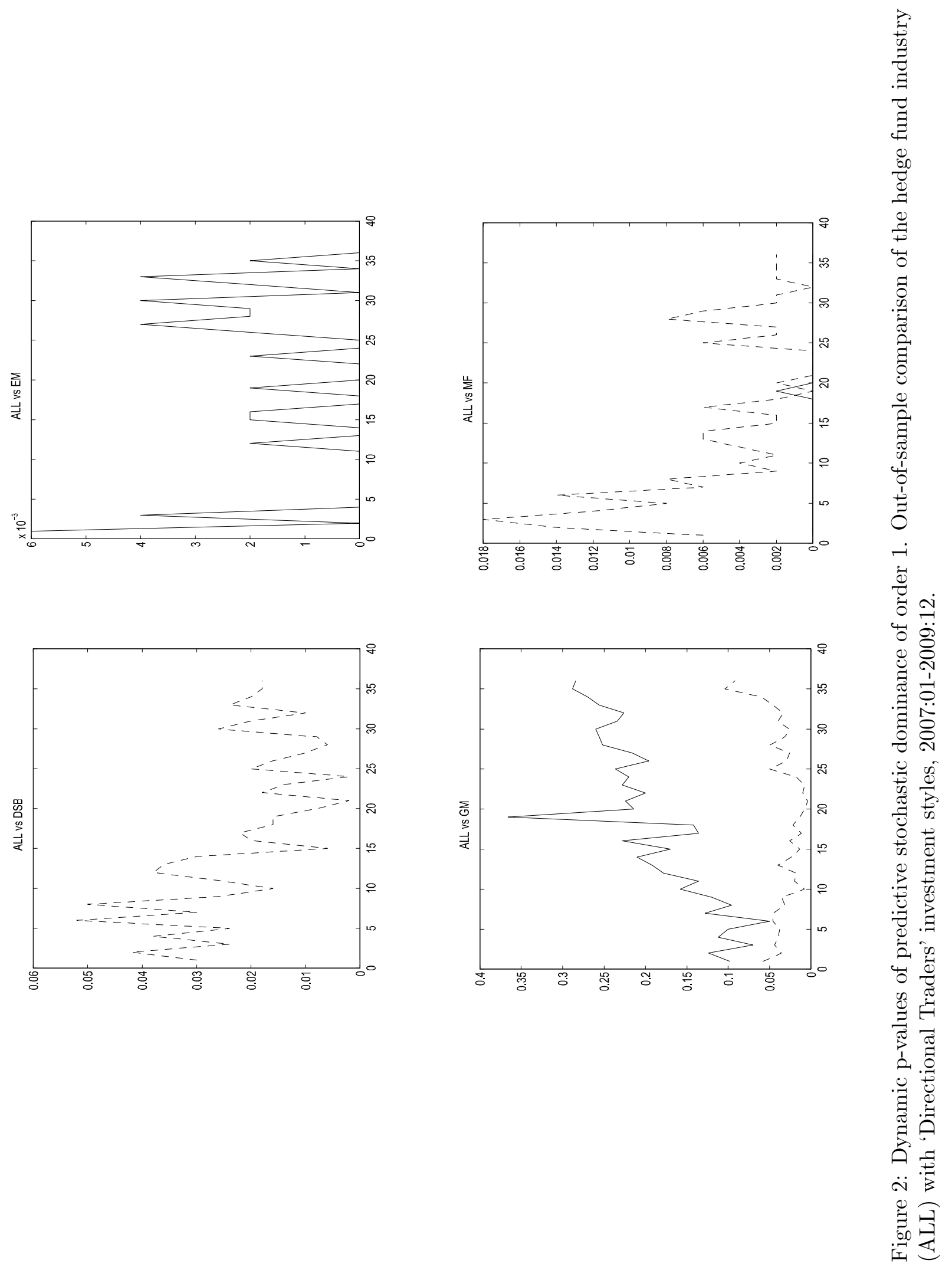

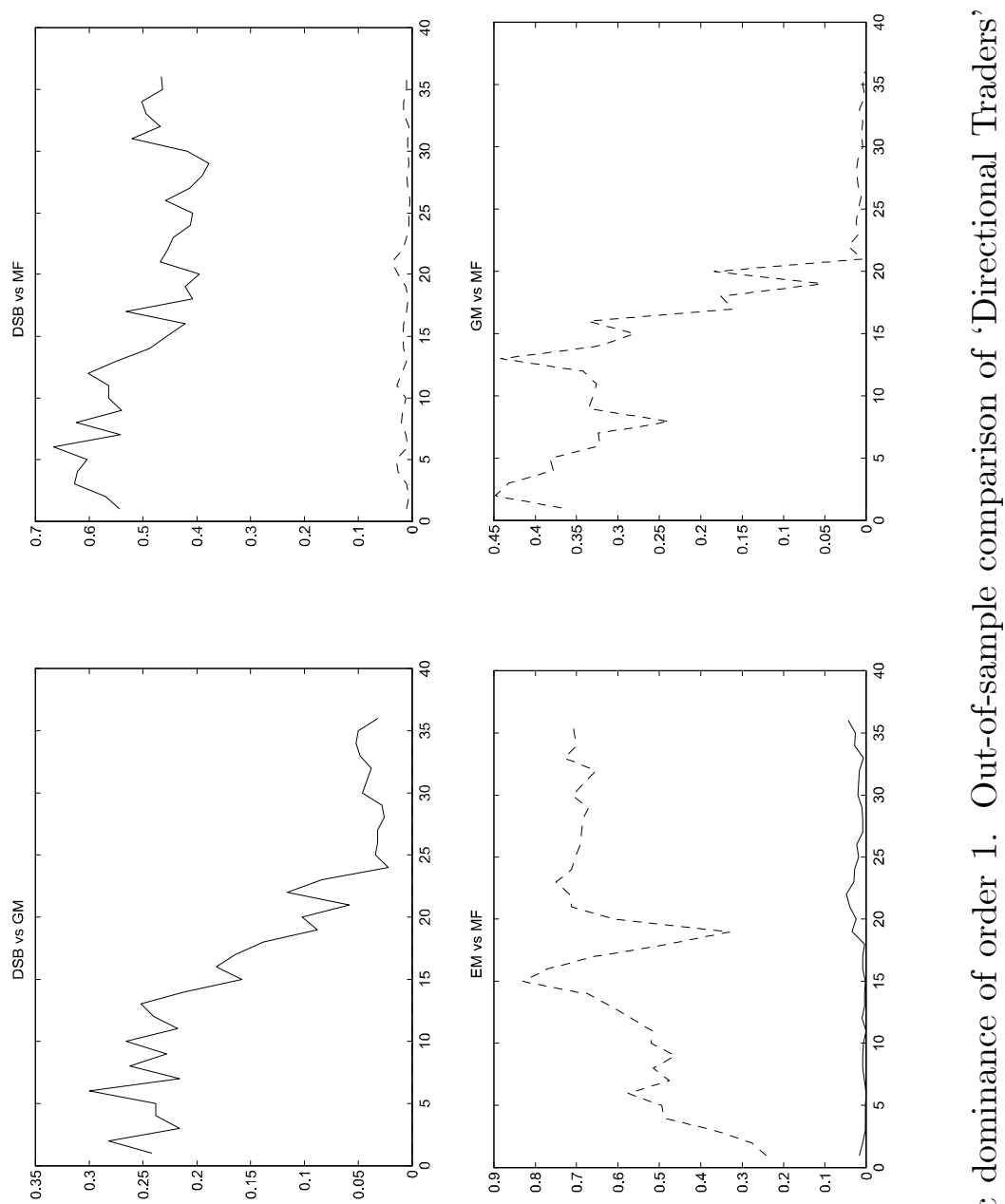

要
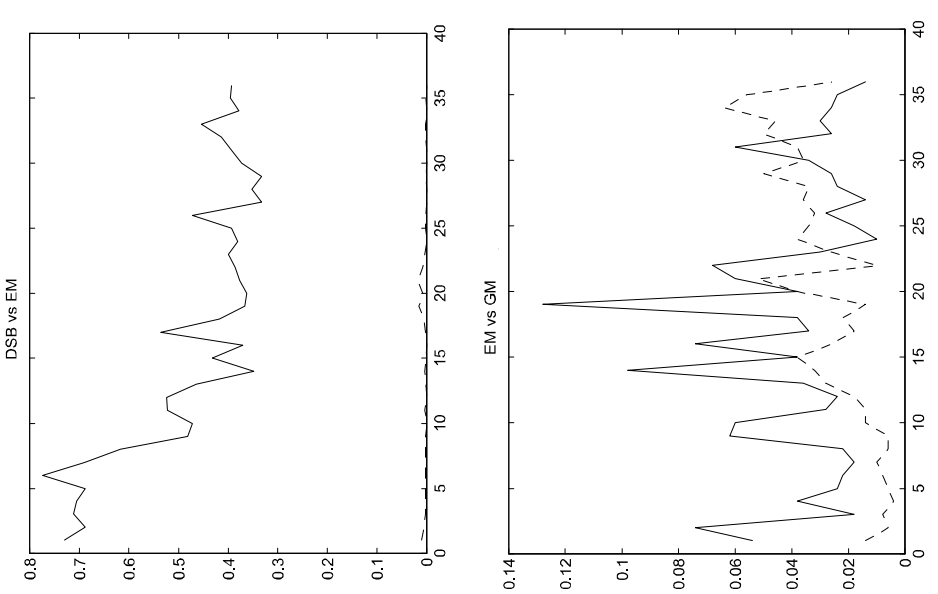

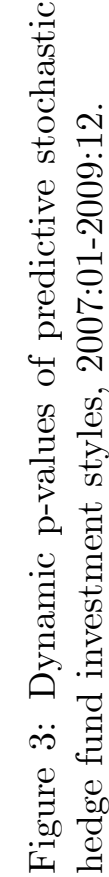



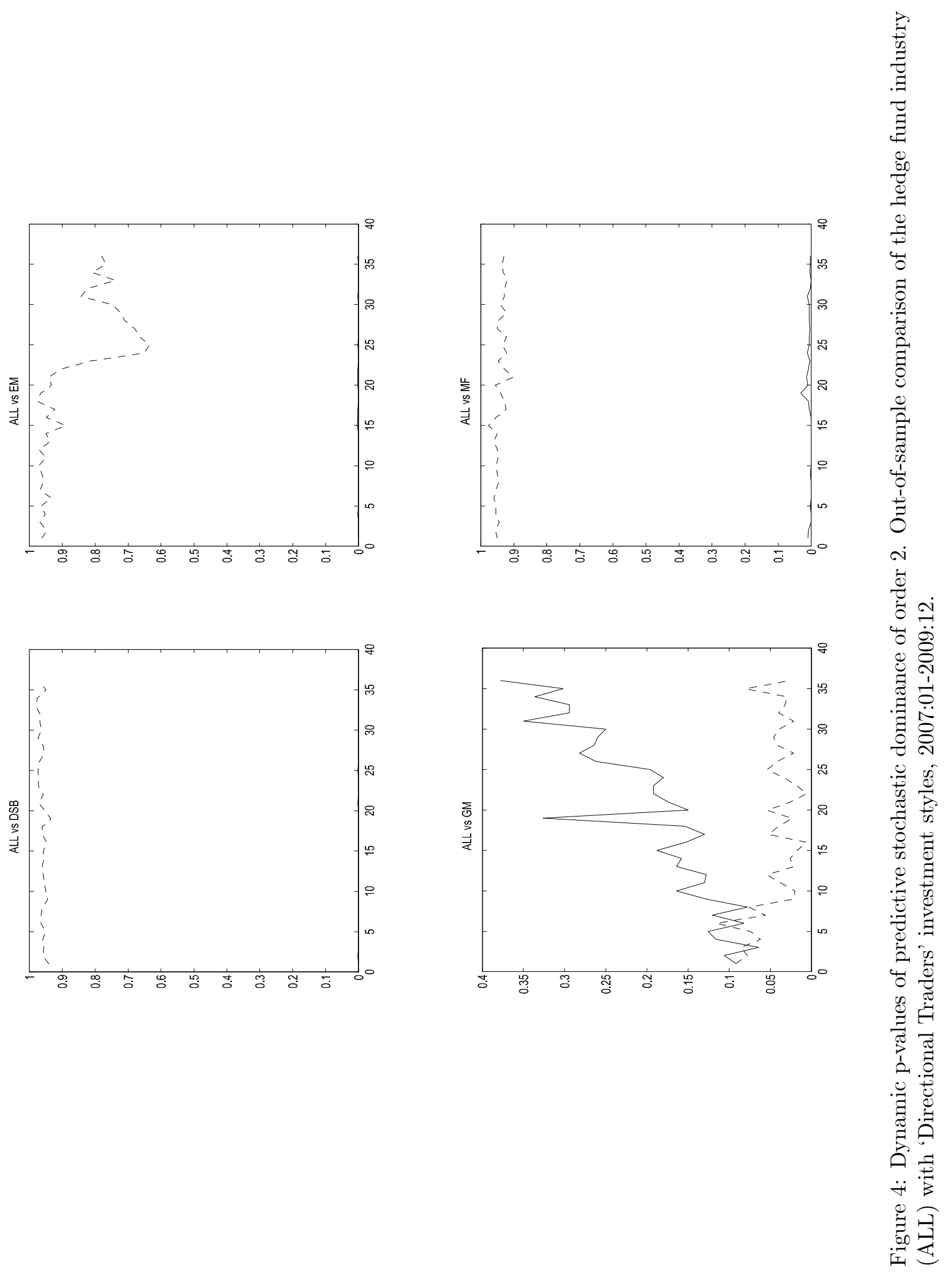

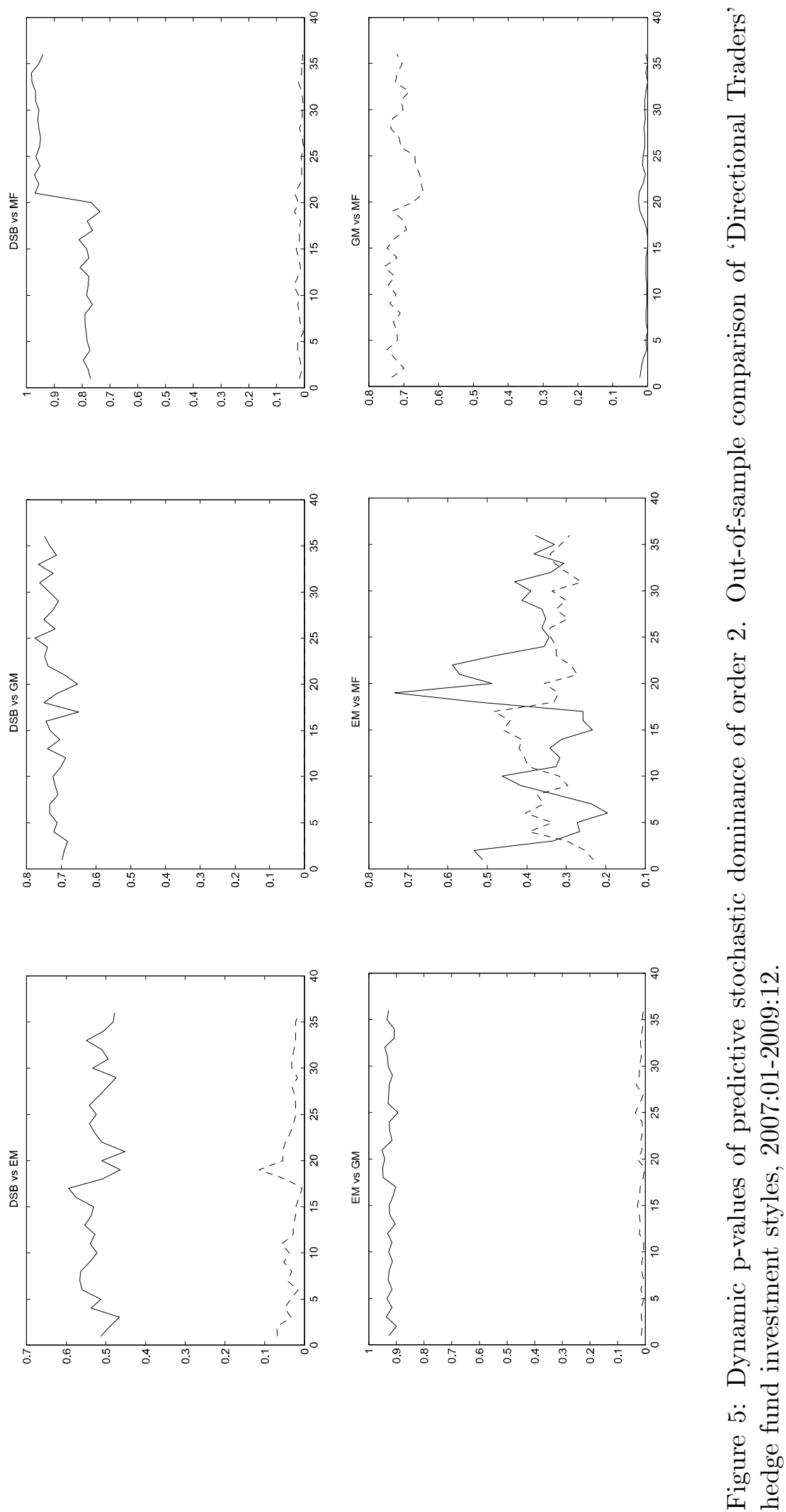